\title{
Information Entropy, Economic Analysis And Financial Reporting
}

Robert A. Nehmer, (Email: rnehmer@campbell.berry.edu), Berry College, Mount Berry, GA

\begin{abstract}
This paper presents a version of entropy theory in economic analysis. This theory is then used to introduce the notion of the social production of value and to critique the idea of transaction. Habermas' theory of communicative action is then employed to understand the production of informational value, a necessary pre-condition to its dissipation. Finally, some suggestions as to how these concepts might be used to influence standards of financial reporting are entertained.
\end{abstract}

\section{Introduction}

l

n the idea of chaos, it seems, lies our last best hope of possibility: of breaking loose the cultural bonds and definitions that constrain our relations to self, to others, to the world, as we must on occasion if we are to remain sane and authentic. Entropy as root metaphor recaptures something of the psychic economy of the walled city, for it tells us that the elements of culture are statistically improbable and temporally parochial pockets of order in a world that marches toward maximum chaos. This vision of incipient chaos is, on the whole, better than the concrete chaos of Armageddon; but as a shadow reality, abstract, invisible, and not quite immanent in our world, it is poor consolation for the loss of nature as Other. [Zencey 1991, 54-5]

Traditional economic analysis serves to collect statistical information on social phenomena and analyzes this information based on paradigms largely derived from the mathematics of classical mechanics. Financial reporting numbers reflect this penchant of data collection and analysis. Recently, the American Institute of Certified Public Accountants held a symposium which examined the directions financial reporting should take to face the challenges of the new century.

Readers with comments or questions are encouraged to contact the authors via email.
One important facet of the situation that the symposium addressed was the fundamental shift in basic technologies from a production and industrial era to an information era.

This paper applies Georgescu-Roegen's concepts of informational entropy and Jürgen Habermas' theory of communicative action to the needs of economic analysis and financial reporting in the twenty first century. It uses these concepts to model the loss of economic value through the loss of information due to entropy in the communicative frameworks employed by human actors. It adds to Georgescu-Roegen's concepts by introducing evolutionary organizational processes which provide one component in the production of value and information. Both the production of value/information and its eventual and inevitable dissipation are seen to be mediated by institutional factors which are produced by social acts. Information value is thus seen to reflect social value and both values are posited to be created by social organizational activities and reduced by entropy. This moves economic analysis away from a purely material notion of the production of value. The paper begins with a presentation of entropy theory in economic analysis. This theory is then used to introduce the notion of the social production of value and to critique the idea of transaction. Habermas' theory of communicative action 
is then employed to understand the production of informational value, a necessary pre-condition to its dissipation. Finally, some suggestions as to how these concepts might be used to influence standards of financial reporting are entertained.

\section{Economic and Information Entropy}

Georgescu-Roegen's penetrating study of the physical law of entropy in The Entropy Law and the Economic Process_serves up much of current economic analysis as econometrics derived from the standard equations of thermodynamics and statistical thermodynamics. This paper can hardly do justice to all of the thought that went into the production of the book, but two basic thrusts are used here: the dependence of economic analysis on statistical thermodynamics as a foundational theory and the idea of the dialectic as a form of life in human analytical processes.

Georgescu-Roegen discusses at some length the formulation of the law of entropy which occurred in the 19th century. The entropy law stipulates that heat energy always moves from a body which has more to a body which has less. This will occur in addition to any of the laws of Newtonian mechanics, or classical thermodynamics, which may be operational in the system as well. An attempt to include entropy calculations in the equation systems of classical thermodynamics led to the formulations of statistical thermodynamics, according to GeorgescuRoegen. Yet he shows in both formal and informal ways how the characterizations of the entropy law in Boltzmann's formulations cannot be said to have captured the principle of entropy. This, of course, has ramifications in the field of physics itself, ramifications which are not lost on all physicists. But Georgescu-Roegen's point is that these problems are not addressed in standard economic analysis, a social science method which takes as its theory a social interpretation of these very same problematic equations. In short, if the metaphor is inapt in its original language, how well would we expect a literal translation to convey the intended meaning?

\section{The Social Production of Value}

The equational systems discussed in the preceding paragraph are examples of what Georgescu-Roegen calls arithmomorphic concepts. As fundamentally non-arithmomorphic concepts, he opposes dialectical concepts to them. The antinomy between One and Many with which Plato, in particular, struggled is well known. One of its roots resides in the fact that the quality of discrete distinction does not necessarily pass from the arithmomorphic concept to its concrete denotations. There are, however, cases where the transfer operates. Four pencils are an "even number" of pencils; a concrete triangle is not a "square. Nor is there any great difficulty in deciding that Louis XIV constitutes a denotation of "king." But we can never be absolutely sure whether a concrete quadrangle is a "square." In the world of ideas "square" is One, but in the world of senses, it is Many. [Georgescu-Roogen 1971, 45] He contrasts to this dialectical concepts, saying that "[T]hey have no arithmomorphic boundaries; instead, they are surrounded by a penumbra within which they overlap with their opposites" [Georgescu-Roegen 1971, 45, emphasis in the original]. The dialectical concept, then, is of a different type than the arithmomorphic and provides for a different mode of analysis. Indeed, dialectical concepts do not stand in an interpretation of a singularity, but are always used in contexts. They bring context and the Other into the discourse by their very existence and are therefore, irreducible to arithmomorphic concepts.

The implications of the dialectic for economic processes are constituted on the very basis of the possibility of the reduction of social, economic events and objects (flows and stocks) to arithmomorphic concepts. Georgescu-Roegen argues that such a reduction is not possible while still maintaining the essentially socially constructed nature of the economic process. His argument concludes with an appeal to the fact that even if it were possible to impose a "rationality" on the actors in economics processes, it would not be desirable to do so. This paper contends that the issue can be brought into clearer focus by evaluating the economic process from the social standpoint of the actors initially, rather than from the 
stance of the equational systems. As such, it introduces the concept of language as the instrument used in the production of value.

Social anthropologists such as Sahlins and Mauss have been investigating the idea of exchange in primitive cultures for several decades. The findings reveal a startling variety of exchange behaviors many of which bear little resemblance to behaviors of rationality and utility maximization which are the standard normative descriptions of modern, capitalistic economies. Exchange behaviors can reflect or define the relative status of the participants, territorial agreements, or even be so routinized that the participants are unable to explain the meaning of the exchange any longer. Of course, the applicability of such exchanges to modern economies can be justly questioned but what is of interest here is the concept that these exchanges are produced by a conscious bargaining or by an unconscious idea of habitual reciprocity. It is the idea of inherent value residing in the particular goods in question which is lacking.

Nissen has written an extremely interesting book about the development of societies in the Near East 4,000 to 11,000 years ago. In it, he explores some of the early evidence on the development of written language in that area. Most of the oldest documents (clay tablets) are concerned with the accounting for various stocks of goods. The very oldest are what one might consider today to be single entry accounts: lists of "product" and an associated "amount." A rather continuous evolution of documents has been found which indicates that a record keeping notion of exchanges was developed after the single entry lists but before there is any significant evidence of true written language through, for example, a body of literature. Indeed, the development of written language recorded in this bedy of evidence is such that it is apparent that these records of exchange significantly influenced both the symbols and the syntax of this written language.

Once a human society is in the position to use written language, however, the well known problem of textual interpretation comes into play. So on the one hand we are in nebulous position as to the interpretation of what the actors believe they have accomplished during an exchange, and on the other hand, if that exchange is recorded, we have the problem of the subsequent interpretation of the record of the exchange. And this is precisely the problem of the double hermeneutic. In this paper the suggestion is made, hardly to "solve" this quandary, but rather to offer an explanation as to what is happening in terms of the informational values that have occurred during the exchange and its subsequent recording and the interpretation of that record.

\section{A Critique of Transaction}

The values ascribed by actors in an exchange, during the act of the exchange, are not entirely circumscribed by an "exchange event frontier" or some notion of the kind. Rather, the exchange can be compared to a single line in a play, which may or may not be instrumental in building a plot, or subplot, or moral to the play as a whole. As such, some exchanges, such as the purchase of a home, have ramifications for further exchanges that might occur in the future, such as the replacement of a furnace or the paying of certain monthly bills. This type of exchange would also be influenced dramatically by past histories of exchanges since these would impact on the size of downpayment available, for instance. Other exchanges, such as the purchase of a dinner, have fewer directly recognizable impacts on future exchanges because our system of accounts does not record the social context of the exchange.

At the time of an exchange such as the house purchase mentioned above, much sensory data is available to any of the participants or indeed to any neutral party present. Very little of this data, however, is recorded. Here we call the recorded data information since it can at least remain potentially useful (or curious) until it becomes illegible. There are two things to note about the above description. First, there has been nothing said about a "meeting of the minds" per se in this transaction. The transaction occurs but the actual psychic valuation issue is not enjoined. Indeed, it cannot be since we have no basis of measuring the parties' individual "utilities" and therefore no model with which to compare those. Secondly, nothing can be said about the meaning 
behind the choice to record certain data, rather, all that could be provided is some type of description of how this choice was arrived at and this description would, as a written record, be subject to interpretation, too.

All of the above is fine, though, and actually accords well with an idea of explaining how entropy impinges on such a system. Shortly after an exchange occurs, it is relatively easy for the participants to reconstruct their perceptions of the valuation issues as they felt them at the time. Furthermore, the interpretation of the record of the exchange is also relatively unproblematic. But, with the onset of time, the remembrance of the particular transaction will fade with the memories of the participants, and finally die as a direct experience. Also, in time the language used to record the information of the exchange, and the language of any description of the model of the recording's intended meanings will lose their primary focus, which came from the recorder his/herself. Both of these factors indicate an increase in entropy in the phenomena of the exchange and in its description (loss of information content).

Forces which will interact upon the two sides of exchange as discussed above (as phenomena and as record) are any of those which will shape the individual actor's consciousness and any which will influence the social norms concerning what will be recorded and what will be the intended (constructed) interpretation of the records. In order for the accounting profession, or any other, to influence this process, it seems clear that the first thing that must be accomplished is a recognition of the basic dynamics involved, which is to say a recognition that although we may wish that we possessed completely satisfactory quantitative models of economic processes, we do not. Furthermore, we should perhaps judge the "happiness" or "utility" of our societies based on the relative lack of social disorder on all levels of society, an observation of action, rather than on summary statistics, an interpretation of symbol.

\section{The Social Production of Informational Value}

This section addresses some issues of communication in group settings using Habermas' theory of communicative action. This is a rich sociological theory of how communication and distortions of communication occur in social communities. According to Habermas, consensus must precede communication. This consensus is based on the validity claims of truth, legitimacy, veracity, and comprehensibility. When all four of these claims are not met, the communication must precede on the level of discourse. Habermas proposes a theory of communicative action (i.e., $1975,1984,1987)$ within which he formulates a framework for understanding the dynamics of capitalist societies. Central to his position is the proposition that for action, motivated by speech acts (Searle, 1969), to be undertaken the validity claims must be satisfied or that these claims could be satisfied if the speaker were called upon to do so. In setting forth this theory, Habermas attempts to engage rationality in carrying out communicative action as opposed to strictly instrumental or "strategic" action. He does so in an attempt to bring to bear the rationalization of modern society in providing emancipatory opportunities.

In order to operationalize some of Habermas' theorizing, this paper concentrates on the linguistic, as opposed to the socio-economic, aspects of the theory of communicative action. Habermas envisions three types of action. The first type is instrumental action which is an action oriented to success in a nonsocial situation. The second type is strategic action which is an action oriented to success in a social setting. The last type is communicative action which is action oriented to reaching understanding in a social situation. Habermas terms all language which occurs while accomplishing these actions as discourse except for a single exception. Language which is used to accomplish communicative action is termed communication.

Habermas says that all communication must be valid (correct) in four ways. First, it must be performed correctly to be comprehensible. This validity claim is often assumed to be true so we will not consider it further here. Second, it must be socially valid, that is, legitimate. This claim is based on the social position of the person who is communicating. That person must be in a 
legitimate social position to perform the specific speech act. Consider the social position one needs to legitimately close a university for a snow emergency. The third validity claim is that of truth, that is, that the statement made is true. The last claim is that the utterance is said sincerely, without irony or malice.

When people are members of a group, their actions and therefore their speech acts may be oriented to a successful pursuit of their own interests rather than achieving an understanding of the interests of other group members. This propensity is used in this study by considering the diversity of interests in groups. As the membership of a group increases in diversity it is expected that the individual group members would tend to rely more on strategic action rather than communicative action.

The work of Habermas implies that discourse is the manifestation of conflicts inherent in the economic system and, as these conflicts intensify, crises arise. Habermas also argues that the acquiescence to the control emanating from enhanced individual "visibility" brought about through advanced technology is not necessarily a fiat accompli. According to the theory of communication action, as long as technology manifestation evaluations can be grounded using traditional norms contained within the socio-cultural system (lifeworld), an emancipatory possibility exists. That is, as long as the validity claims are questioned within the context of communication action, the systemic encroachments can be evaluated and mediated in terms of individual sovereignty as well as instrumental objectives.

In modern societies, instrumental objectives come to dominate, thus depriving individuals of part of their normal sovereignty. The individual is not subjectively involved, having little power to actively influence the course of events. Specifically, social systems, and the crises therein, "are not produced through accidental changes in the environment, but through structurally inherent system imperatives that are incompatible and cannot be hierarchically integrated." This suggests that in order to understand society an appreciation of the "structurally inherent sys- tem-imperatives" is needed.

In a recent paper, Nehmer manipulated the membership of student groups. Students enrolled in a masters level accounting program were first given a Meyers-Briggs type personality profile. The results of profile were used to form four groups consisting of similar and dissimilar individuals. The students were also given an accounting information systems case to read and were asked prepare an individual solution. The students were asked to use Checkland's Soft System Methodology (SSM) as a framework to solve the case. Then they were asked to get together and solve the case as a group. The results were analyzed by comparing the group solutions to the individual solutions. Information loss, a solution mentioned by a student individually but not mention by his or her group, and information production, a group result not obtained by any individual of that group, were evaluated. While the results are tentative, they do allow group dynamics to be explained in a theoretical framework. It appears as though groups with little personality diversity tend to loose much information quickly while those groups with too much diversity may be coopted by a strong individual whose solution dominates the group's solution. A pre-test response by students as to what they saw as the problem in the case was also solicited and was found to provide higher explanatory power than personality types. Groups whose members held similar views as to what the problem situation was, such as who was the "good guy," who was the "bad guy," and what problem needed to be addressed tended to produce the most new solutions to the case.

\section{Conclusions}

In terms of an information theoretic perspective of these ideas, it is perhaps time that we stopped using the thermodynamic equation specifying entropy increase as a direct metaphor of information loss. First, since there is no reason to believe that these equations, in their multitudinous forms, are ever unproblematical for physicists themselves, it seems the better part of discretion to avoid any equivalencing of the two. Secondly, the social system in all of its constituent 
parts, along with all physical systems, is subject to the law of entropy. There is no basis for pointing at any particular social behavior and claiming that it is driven exclusively by the law of entropy, or described perfectly thereby, without consideration of every other physical law that must be simultaneously operating at the exact time of the behaviors in question. Information as used here is a socially constructed technique for recording selective impressions of an event in symbols. Those symbols must then be re-interpreted and the ability to do so and to derive the intended meaning is subject to a type of entropy. It is not the intention of this paper to suggest that this is explicitly arithmomorphic.

The results of Nehmer's study seem to indicate that group creativity and the eliciting of a communicative action response rather than a strategic response is more influenced by the relative homogeneity of the group's worldviews concerning the task, rather than the group members' personality types. This has some intuitive appeal since it indicates that the group members were more concerned with task completion than with personality conflicts. The completion of the task at hand is also an action in Habermas' sense, so manipulations of worldview in group composition would seem to come closer to investigating the difference between strategic and communicative forms of action in group decision making. Considering further directions for this type of research might seem to be looking for the next turtle. The research presented here investigates how group composition alters the possibility of communicative action which in turn affects the evolution of common worldviews through common understandings.

\section{Suggestions for Future Research}

Future research could be accomplished in three areas. The first area is in the study of transacting. This paper has speculated somewhat on transacting in group settings. Further research is possible by broadening the idea of group into one of culture and then multi-cultural settings. This research would be in the worldview context as discussed above. It could also involve a decision task which was more related to economic transacting, rather than group consensus of a business system problem. A second area of research would investigate applying other economic theories in accounting contexts. A potentially interesting variation of this would apply the economic theory from a systems perspective. The third area of further research lies in developing formalizations of information value and information entropy in business settings. Possible implications of this avenue of investigation accrue in the areas of data archiving and aggregate value versus detailed value.

The author would like to thank Barbara Merino for her consistent encouragement. The author appreciates the supportive environment provided by the Campbell School of Business at Berry College.

\section{References}

1. Adams, J. (ed.), Institutional Economics: Essays in Honor of Allan G. Gruchy, Martinus Nijhoff Publishing, Boston, 1980.

2. Cheal, D., The Gift Economy, Routledge, New York, 1988.

3. Commons, J., Institutional Economics, MacMillan, New York, 1934.

4. Dorfman, J., C. E. Ayres, N. Chamberlain, S. Kuznets and R. A. Gordon, Institutional Economics: Veblen, Commons, and Mitchell Reconsidered, University of California Press, Berkeley, 1963.

5. Flood, Robert L. and Ewart R. Carson, Dealing with Complexity, Plenum, New York, 1993.

6. Gabel, C., Analysis of Prehistoric Economic Patterns, Holt Rinehart and Winston, New York, 1967.

7. Georgescu-Roegen, N., The Entropy Law and the Economic Process, Harvard University Press, Cambridge, 1971.

8. Georgescu-Roegen, N., Energy and Economic Myths: Institutional and Analytical Economic Essays, Pergamon Press, New York, 1976.

9. Gordon, W., Institutional Economics: The Changing System, University of Texas Press, Austin, 1980.

10. Gorz, Andre, Critique of Economic Rea- 
son, translated by Gillian Handyside and Chris Turner, Verso, New York, 1989.

11. Gruchy, A. G., The Reconstruction of Economics, Greenwood Press, New York, 1987.

12. Habermas Jurgen, Legitimation Crisis, translated by Thomas McCarthy, Beacon Press, Boston, 1975.

13. Habermas, Jurgen, The Theory of Communicative Action: Reason and the Rationalization of Society (vol. 1), translated by Thomas McCarthy, Beacon Press, Boston, 1984.

14. Habermas, Jurgen, The Theory of Communicative Action: Lifeworld and System: A Critique of Functionalist Reason (vol. 2), translated by Thomas McCarthy, Beacon Press, Boston, 1987.

15. Habermas, Jurgen, The Philosophical Discourse of Modernity, translated by Frederick Lawrence, MIT Press, Cambridge, 1987.

16. Jackson, Michael C., Systems Methodology for the Management Sciences, Plenum, New York, 1991.

17. Katz, J. J., Language and Other Abstract Objects, Rowman and Littlefield, Totowa, New Jersey, 1981.

18. Lakoff, George and Mark Johnson, Metaphors We Live By, University of Chicago Press, Chicago, 1980.

19. Mattessich, Richard, Accounting and Analytical Methods, Irwin, Homewood, Illinois, 1964.

20. Mauss, M., The Gift, Norton, New York, 1967.

21. Nissen, H. J., The Early History of the Ancient Near East: 9000 - 2000 B.C., The University of Chicago Press, Chicago, 1983.

22. Putnam, Linda L. "The Interpretive Perspective: An Alternative to Functionalism," Communication and Organizations: An Interpretive Approach, Linda L. Putnam and Michael E. Pacanowsky (eds.), SAGE, Beverly Hills, California, 1983.

23. Rockmore, T., Habermas on Historical Materialism, Indiana University Press, Bloomington and Indianapolis, 1989.

24. Sahlins, M., Stone Age Economics, Aldine
Publishing Co, New York, 1972.

25. Veblen, T., The Theory of Business Enterprise, Scribner, New York, 1927.

26. Veblen, T., The Vested Interests and the Common Man, Viking Press, New York, 1964 [c1919].

27. Veblen, T., The Theory of the Leisure Class, Penguin Books, New York, 1979.

28. Zencey, E., "Entropy as Root Metaphor," Beyond the Two Cultures: Essays on Science, Technology, and

29. Literature, Slade, J. W. and J. Y. Lee (eds.), Iowa State University Press, Ames, 1990.

30. Zencey, E., "Some Brief Speculations on the Popularity of Metaphor and Symbolic Activity," Metaphor and Symbolic Activity, Vol. 6, No. 1, pp. 47-56, 1991. 
